\title{
Confluent Volumetric Visualization of Gyrokinetic Turbulence
}

\author{
George Stantchev, Derek Juba, William Dorland, and Amitabh Varshney
}

\begin{abstract}
Data from gyrokinetic turbulence codes are often difficult to visualize due their high dimensionality, the nontrivial geometry of the underlying grids, and the vast range of spatial scales. We present an interactive visualization framework that attempts to address these issues. Images from a nonlinear gyrokinetic simulation are presented.
\end{abstract}

Index Terms-Gyrokinetics, plasma turbulence, visualization.

G YROKINETIC simulations of tokamak turbulence are widely used to interpret experimental data. The level of realism in modern gyrokinetic codes is very high-so high, in fact, that it is nearly as difficult to understand and interpret the simulation results as it is to work directly with experimental data. One of the main problems is that the data sets, such as those shown here, are typically of dimension greater than three. In addition, many gyrokinetic simulations take place in a flux-tube following coordinates whose geometry is fairly nontrivial and whose size with respect to the enveloping tokamak device is small. Moreover, the very nature of turbulence implies the existence of structures at various scales, which turns coherent graphical representation of data into a challenging problem.

A gyrokinetic simulation describes the time evolution of the 5-D probability distribution function(s) for a few plasma species (typically, one to five). Three of these dimensions are spatial, and the other two correspond to velocity space variables. Visualizing such a data set directly is clearly impossible. A traditional diagnostic quantity is the electric potential $\phi$ whose fluctuations capture the patterns of turbulence present in the system.

One of the most compelling techniques for visualizing 3 -D scalar fields is direct volume rendering. It gives a global qualitative view of the entire data set, providing a fast insight into spatial patterns and correlations. However, direct volume rendering works best for scalar fields with high degree of regularity, such that occlusion and cluttering can be eliminated by filtering easily identifiable value ranges. This is not the case with the electric potential, or other turbulent scalar fields that arise in this context, which exhibit fluctuations on a variety of scales.

To deal with this problem, we focus on derived diagnostic quantities, which are both physically meaningful and suitable

Manuscript received December 2, 2007.

The authors are with the University of Maryland, College Park, MD 20742 USA (e-mail: gogo@math.umd.edu).

Digital Object Identifier 10.1109/TPS.2008.924509 for direct volume rendering. One such diagnostic is the heat flux, which is calculated as a velocity space integral

$$
Q=\int f E \frac{\partial\langle\phi\rangle}{\partial y} d \mathbf{v}
$$

where $f$ is the gyrokinetic probability distribution function, $E$ is energy, and $\langle\phi\rangle$ denotes the gyroaveraged electric potential. The values of $Q$ are used to identify regions where the heat flux satisfies a certain condition, for instance, specified by a given range. Within each region of interest, we map the values of a turbulent field, such as the electric potential. Thus, information from two fields with distinct spatial characteristics is convolved into a single coherent visual representation.

The data presented here are taken from an electrostatic gyrokinetic simulation of the Cyclone [1] benchmark case for ion temperature-gradient-driven turbulence in a tokamak. The spatial simulation domain is $\left(n_{x}, n_{y}, n_{z}\right)=(96,96,64)$, where $n_{x}$ and $n_{y}$ are the number of grid points in the plane locally perpendicular to the background magnetic field and $n_{z}$ is the number of grid points along the magnetic field. There are 768 velocity-space grid points at every spatial grid, for a total mesh point count of about half a billion. As is standard in the Cyclone benchmark, there is one gyrokinetic ion species, and the electrons are taken to have a Boltzmann response. The code used to generate this data is the GS2 code [2], [3].

The images on Fig. 1 show "confluent" volumetric rendering (CVR) of the heat flux and the electric potential in the computational domain. Heat-flux values are used in the opacity transfer function, in effect, defining a set of visible spatial structures inside the volume. Electric-potential values are used in the color transfer function, which controls how color is applied to visible pixels. In our setup, we are looking at a collection of island regions where heat flux is higher than a certain cutoff value. The image on Fig. 2 shows the geometry of the flux-tube domain and features a blown-up region that corresponds to a small ball around the center of the computational domain. Note that, for aesthetics reasons, the partial toroidal shell shown as a reference here is taken from a different tokamak configuration.

Shown here are data derived from the late linear phase of the simulation, when the linearly unstable modes are at high amplitude and are strongly interacting, but before the nonlinearly generated flows and eddies have reached high amplitude. The amount of heat being transported at this instance is quite large (comparable to the steady-state turbulent value), despite the small spatial filling factor. A conventional diagnostic would show "streamers" at this point in time. We are using the new CVR diagnostic to develop intuition about the relationship between the $E \times B$ flows (along contours of constant potential) and the radial heat flux. 


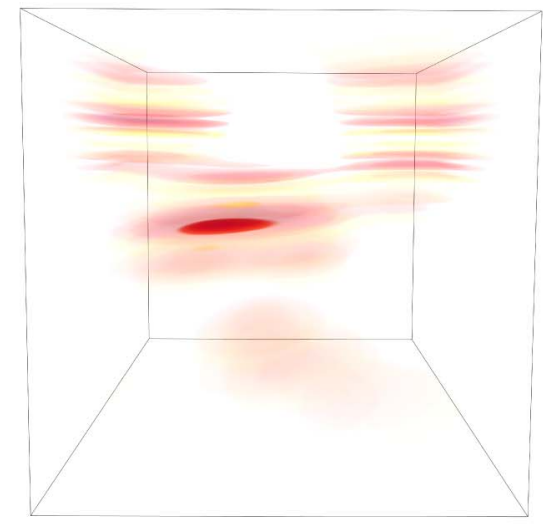

(a) radial-poloidal view

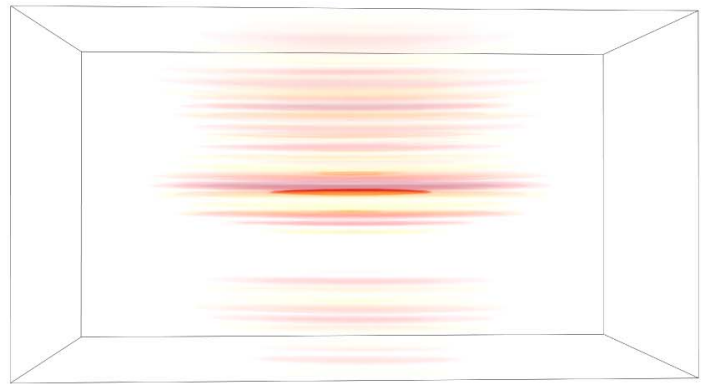

(b) poloidal-toroidal view

Fig. 1. Confluent volume rendering of the computational domain. Visible structures indicate zones of high heat flux. Color represents values of the electric potential.

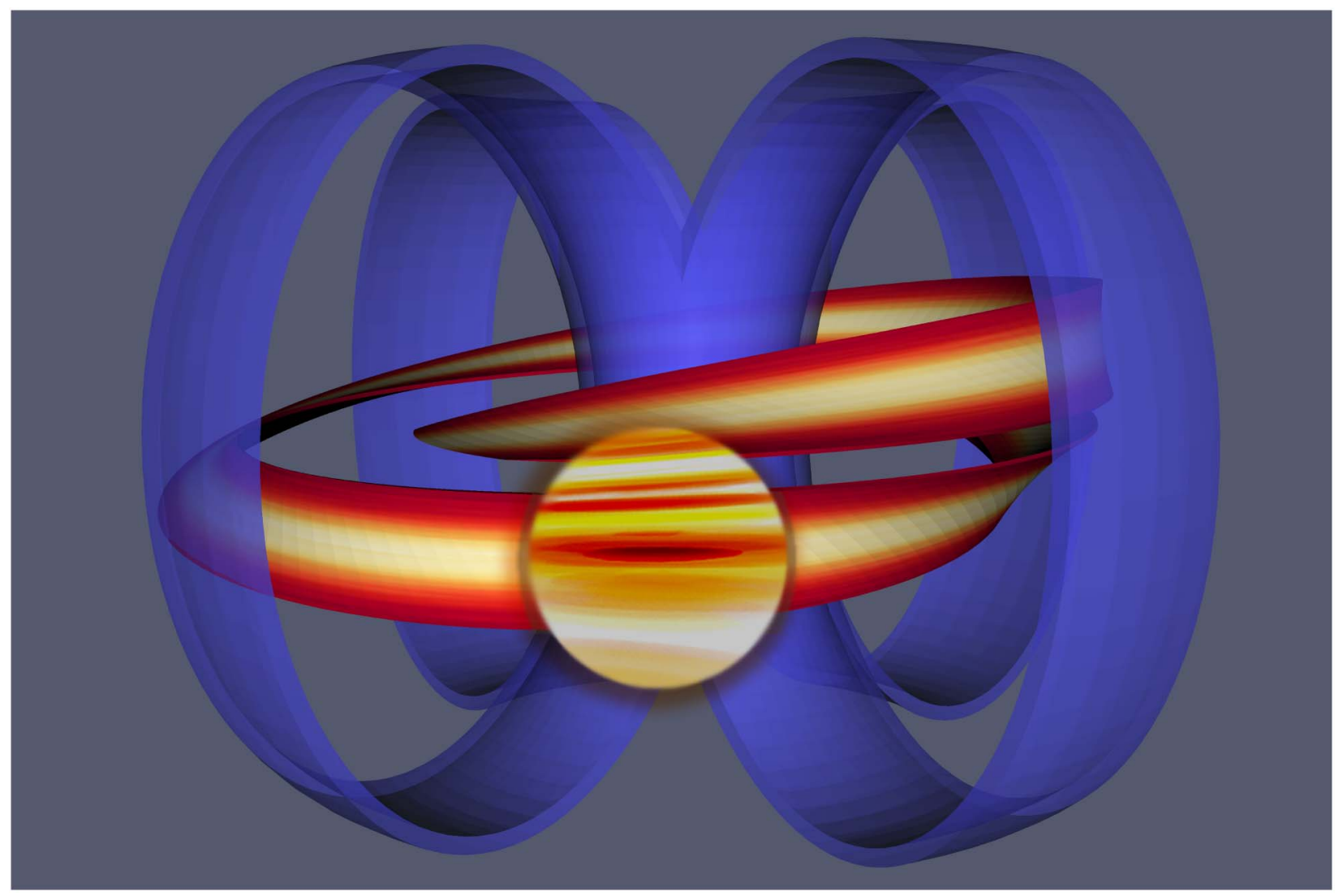

Fig. 2. Flux-tube domain in a toroidal device. The outer shell of the flux tube is rendered as a surface with color controlled by the values of the electric potential alone. The circular inset in the middle shows a magnified view of a small spherical region inside the flux tube visualized via confluent volume rendering.

\section{REFERENCES}

[1] A. M. Dimits, G. Bateman, M. A. Beer, B. I. Cohen, W. Dorland, G. W. Hammett, C. Kim, J. E. Kinsey, M. Kotschenreuther, A. H. Kritz, L. L. Lao, J. Mandrekas, W. M. Nevins, S. E. Parker, A. J. Redd, D. E. Shumaker, R. Sydora, and J. Weiland, "Comparisons and physics basis of tokamak transport models and turbulence simulations," Phys. Plasmas, vol. 7, no. 3, pp. 969-983, Mar. 2000.
[2] W. Dorland, F. Jenko, M. Kotschenreuther, and B. N. Rogers, "Electron temperature gradient turbulence," Phys. Rev. Lett., vol. 85, no. 26, pp. 5579-5582, Dec. 2000.

[3] M. Kotschenreuther, G. Rewoldt, and W. Tang, "Comparison of initial value and eigenvalue codes for kinetic toroidal plasma instabilities," Comput. Phys. Commun., vol. 88, no. 2, pp. 128-140, Aug. 1995. 